\title{
Range space super spherical cap discriminant analysis
}

\author{
Jihong Pei ${ }^{\mathrm{a}}$, Hongguang Fan ${ }^{\mathrm{a}, *}$, Lijuan $\mathrm{Pu}^{\mathrm{a}}$ \\ ${ }^{a}$ College of Information Engineering, Shenzhen University, Shenzhen, 518060, China
}

\begin{abstract}
To overcome the separability problem caused by sample fusion in the process of sample vectors normalization, this paper presents a unit super spherical cap discriminant analysis in the range space of the total scatter matrix. It is proved that the unit super spherical cap model can maintain the topological invariability of the structural characteristics of sample vectors. Furthermore, a sufficient condition is derived for improving the separability of sample data under the proposed model. The proposed algorithm projects sample data to the range space of the total scatter matrix, and then adds one dimension to each sample of the range space and nonlinearly maps it on the surface of the unit super spherical cap. We put forth a new classifier called the "spherical inner product nearest neighbor classifier" for the transformed data. It is designed for the deviation problem of the discriminant vector and the separability problem caused by sample vectors normalization when different sub-classes are located in different low-dimensional subspaces or manifolds. Experimental results on different databases show that our method outperforms other methods in terms of recognition accuracy and numerical stability.
\end{abstract}

Keywords: Fisher linear discriminant analysis; separability problem; super spherical cap discriminant analysis; range space.

\section{Introduction}

Fisher linear discriminant analysis (FLDA) [1] is one of the most popular feature extraction methods. It has been successfully applied to various pattern classification problems. However, FLDA adopts the betweenclass scatter matrix as the maximum metric. This may limit the maximal dimensions of the extracted feature space to $\mathrm{C}-1$, where $\mathrm{C}$ is the number of classes of training samples. If the dimensions of the feature space are below $\mathrm{C}-1$, the close classes in the original space will be merged. This is the so-called separability problem [2]. In addition, a basic assumption of FLDA is that the class distribution of samples is homoscedastic. This assumption is impossible in some practical applications [3]. This is known as the heteroscedasticity problem in pattern classification.

In the past, many feature extraction methods have been proposed for pattern recognition [4]-[11]. An enhanced fisher discriminant criterion (EFDC) was proposed in [9]. EFDC combined the intra-class variation with the Fisher discriminant criterion to construct an efficient dimensionality reduction function. The main advantage of this method was stable intra-class representation and it provided very useful discriminant information for classification. Considering local geometrical structure information also includes the local inter-class pattern variation, Di Zhang et al.[10] proposed a complete global-local LDA method (CGLDA) to incorporate local similarity information, local intra-class pattern variation, and local inter- class pattern variation. To effectively utilize global and local geometrical structures, a joint global and local-structure discriminant analysis (JGLDA) [11] was presented to reduce dimension. These methods showed good performance in some databases. To overcome the singular problem of matrix when calculated projection vectors, PCA [12] was first used to make the matrix become nonsingular. However, some potential discriminatory

\footnotetext{
* corresponding author

Email address: 2120090206@email.szu.edu.cn (Hongguang Fan)
} 
information, within the small principal components, was lost in the PCA transformation step [13]. An optimal FLD algorithm (OFLD) for feature extraction was presented in [13]. All the small components and discriminatory information were reserved after K-L transform. In the process of extracting a feature, we should think about how to reduce the loss of effective discriminant information and maintain the stability of the algorithm.

According to the Fisher discriminant criterion, the null space of the total scatter matrix $S_{T}$ does not contain discriminant information, but the range space of the total scatter matrix contains two types of effective discriminant vectors. The first type of vector satisfies the conditions that the within-class distance equals zero and the between-class distance is positive. The second type of vector satisfies the condition that the within-class distance is greater than zero. In fact, the two types of discriminant vectors, respectively, span the null space and range space of the within-class scatter matrix $S_{W}$. Using these discriminant vectors, many feature extraction methods have been proposed in the existing literature. Jing Jin et al. [14] proposed an approach based on Fisher discriminant null space (FDNS) for decomposition of mixed pixels in hyperspectral imagery. It can effectively solve the endmember spectral variability problem, which results in reducing the accuracy of the decomposition of mixed pixels. Actually, the method extracted the first kind discriminant vectors in the range space of $S_{T}$. H.Cevikalap et al. [15] exploited discriminative common vectors (DCV) based on a variation of Fisher's linear discriminant analysis for face recognition. To increase the calculation efficiency, a common vector for each individual class was obtained by removing the differences of the samples in each class. In fact, the common vectors represented the first type of feature vectors. N.Vaswani et al. [16] employed a principal component null space analysis (PCNSA) method for image and video classification. It can mainly solve the problem of different classes having unequal and nonwhite noise covariance matrices. The algorithm extracted the approximate null space in the principal components subspace of the entire data and had a very good inhibitory effect for noise. PCNSA did not take into account the small variance projection directions of the original data space, and these projection directions may have contained rich discriminant information because they had good aggregation for some databases [17]. M.L Zhu et al. [18] presented a classification algorithm, pruning noisy bases discriminant analysis (PNBDA), which extracted strong correlation of the feature vectors in the within-class scatter matrix and between-class scatter matrix. The method did not directly use the Euclidean distance metric but efficiently used to prune the noisy basis of data. However, the weak correlation of the feature vectors may also contained some discriminant information for some databases.

To effectively decrease the loss of discriminant information, scholars have put forth various other methods. The generalized K-L expansion (GKLE) algorithm [19] extracted two types of eigenvectors of the withinclass scatter matrix at the same time. The extracted eigenvectors were associated with zero eigenvalues and nonzero eigenvalues, respectively. Important discriminant information exists in the null space of the withinclass scatter matrix [20]-[22]. A two-stage linear discriminant analysis algorithm was presented that extracted the range space and null space information of scatter matrices [23]. When the number of training samples is far smaller than data dimensions, the within-class scatter matrix and the between-class scatter matrix could be singular. In [23], the regularization technique was utilized to compute the eigenvectors of $S_{W}^{\prime}-1 S_{B}$ and $S_{B}^{\prime-1} S_{W}$, where non-singular matrix $S^{\prime}$ was the approximation of singular matrix $S$. The recognition accuracy of this algorithm based on the accuracy of matrix approximation. An optimal symmetrical null space (OSNS) of the Fisher discriminant was presented for feature extraction and pattern recognition in [24]. The OSNS algorithm not only extracted the null space and its orthogonal complement space of the between-class scatter matrix, but also obtained the null space and its orthogonal complement of the withinclass scatter matrix. A dimensionality reduction method by integrating sparse representation and fisher criterion was proposed in [8] and this method had very good computational efficiency. In order to fully utilize different features, a feature fusion strategy (FFS) was proposed in [25]. It nonlinearly combined Euclidean and manifold structures and automatically determined the optimal parameters. A supervised kernel construction method (SK-PCA) was presented in [26] based on nonlinear mapping idea. To improve the recognition accuracy, the class label information was incorporated into the kernel matrix in SK-PCA. In the most of above methods, obtaining part of the discriminant vectors from the null space can effectively reduce the loss of discriminant information. However, the eigenvectors associated with zero eigenvalues 
maybe unstable, which is caused by small variations of the number of training samples.

The authors presented a range space hyperspherical discriminant analysis (RHDA) [27]. Under this hypersphere-mapping model, the mapped data maintains the same direction and structural characteristic as that of the original data. So it has good recognition accuracy. However, there exist two problems whcih we need deep consideration for the hypersphere-mapping method. Firstly, the hypersphere in n-dimensional space is (n-1)-dimensional. After the n-dimensional sample points are projected onto a unit hypersphere, the sample points are densely distributed on the unit hypersphere and the dimensions of sample space are reduced from n-dimension to (n-1)-dimension. These are the basic reasons which lead to sample fusion exists in the projection process of sample vectors unitization. In fact, when sample points are projected onto the unit hypersphere, all the sample vectors which have the same direction with spherical center will be overlapped. This means the sample fusion problems maybe occur frequently in the process of sample vectors normalization for some databases. Secondly, in the process of sample vectors normalization, the deviation of the spherical center may also affect the number of overlapping samples. For the same data, choose different training samples and spherical center, the number of overlapping samples is also different. If there is a large difference of the sample number between different classes, the spherical center may be partial to the class that has many more samples. Considering the above problems will affect the design of our classifiers and classification precision, we propose a new method called the range space super spherical cap discriminant analysis (RSCDA) to overcome the above problems. The super spherical cap in $(n+1)$-dimensional space is an $n$-dimensional manifold. By adding one dimension to every sample, the super spherical cap in $(n+1)$ dimensional space can keep the dimensions of transformed space unchanged, which can effectively overcome the sample fusion problem in the process of sample vectors normalization. "The classifier of our algorithm is based on the angle cosine distance. It would classify a test sample into class $i$ only if the test sample located in the internal of some small spherical cap that uses the $i$ th class sample as a spherical center, with some threshold as a radius. It shows via theoretical analysis that the data in the surface of the unit super spherical cap not only keeps the structural information of the samples stable, but also avoids the overlapping phenomenon.

This paper is organized as follows. In section 2, we introduce the range space hypersphere model. In section 3, we propose the range space super spherical cap model and analyze its nature. Section 4 gives a new classifier called the "spherical inner product nearest neighbor classifier." Section 5 summarizes the steps of our algorithm. In section 6 , the experiments are performed on three different databases and compared to other methods. The conclusion and future directions are discussed in Section 7.

\section{Related work}

Let the training set be composed of $N$ pairs of $\left(\mathbf{x}_{1}, y_{1}\right),\left(\mathbf{x}_{2}, y_{2}\right), \ldots,\left(\mathbf{x}_{N}, y_{N}\right)$, where $\mathbf{x}_{i}$ denotes the ndimensional training sample and $y_{i} \in\{1,2, \ldots, C\}$ is the corresponding class label. The training samples set $X=\left\{\mathbf{x}_{1}, \mathbf{x}_{2}, \ldots, \mathbf{x}_{k}, \ldots, \mathbf{x}_{N}\right\}$ is also represented by $C$ class sample sets $X=X_{1} \cup X_{2} \cup \ldots \cup X_{C}$, where $X_{i}=\left\{\mathbf{x}_{1}^{i}, \mathbf{x}_{2}^{i}, \cdots, \mathbf{x}_{k}^{i}, \cdots, \mathbf{x}_{N_{i}}^{i}\right\}, i=1,2, \cdots, C$ and $N_{i}$ is the sample number of class $i$. Obviously, the total sample number is $N=\sum_{i=1}^{C} N_{i}$ samples. Then the total scatter matrix and between-class scatter matrix are defined as [28]

$$
\mathbf{S}_{T}=\sum_{k=1}^{N}\left(\mathbf{x}_{k}-\mu_{0}\right)\left(\mathbf{x}_{k}-\mu_{0}\right)^{T} ; \mathbf{S}_{B}=\sum_{i=1}^{C} N_{i}\left(\mu_{i}-\mu_{0}\right)\left(\mu_{i}-\mu_{0}\right)^{T}
$$

where $\mu_{i}$ and $\mu_{0}$ are, respectively, the mean of the samples in the $i$ th class and the total mean of all samples.

In the high dimensional space, when the dimension of the sample space is larger than the number of samples in the training set, the range space $V_{T}^{\perp}$ and the null space $V_{T}$ of $S_{T}$ would arise at the same time. Let $\left(\lambda_{l}, \phi_{l}\right)$ be the $l$ th pair of the eigenvalue and eigenvector of $S_{T}$. Without loss of generality, suppose $\lambda_{1} \geq \cdots \geq \lambda_{t_{T}}>\lambda_{t_{T}+1}=\cdots=\lambda_{n}=0$, then $V_{T}^{\perp}=\operatorname{span}\left(\phi_{1}, \ldots, \phi_{t_{T}}\right)$. This means that $V_{T}^{\perp}$ is spanned by the eigenvectors corresponding to the positive eigenvalues $\lambda_{1}, \ldots, \lambda_{t_{T}}$. A sample $\mathbf{x}$ is projected onto the range 
space by a linear transformation $\overline{\mathbf{x}}=\boldsymbol{\Phi}^{T} \mathbf{x}$, and the variance on each dimension of $\overline{\mathbf{x}}$ is greater than zero. The range space $V_{T}^{\perp}$ excludes the directions for which the within-class distance and the between-class distance equal zero. Thus, there is no discrimination information loss in the process of dimensional reduction.

The sample vector distribution contains its unique pattern characteristic in high dimensional space. The unitization of the sample vector can represent the structure of the sample. Suppose that $\tilde{\mathbf{x}}=\overline{\mathbf{x}} /\|\overline{\mathbf{x}}\|$, where $\overline{\mathbf{x}} \in V_{T}^{\perp}$ and $\|\overline{\mathbf{x}}\|$ denotes the Euclidean norm of $\overline{\mathbf{x}}$. The square of each element of $\tilde{\mathbf{x}}$ can stand for the proportion of the corresponding element of $\overline{\mathbf{x}}$. For example, the unitized vector of $\mathbf{a}=[3,4]$ is $\tilde{\mathbf{a}}=[0.6,0.8]$. The ratio of the first element and the second element in a are, respectively, $(0.6)^{2}=0.36$ and $(0.8)^{2}=0.64$. The proportion of each component in the sample vector is called the structural characteristic of this vector. It is worth noting that the samples in the same class have similar vector structures and those in different classes have different vector structures. Hence, we can classify the samples according to the different structural information.

The first step of range space hyperspherical discriminant analysis (RHDA) can be described by the formula $\tilde{x}=\boldsymbol{\Phi}^{T}\left(\mathbf{x}-\mu_{0}\right) /\left\|\boldsymbol{\Phi}^{T}\left(\mathbf{x}-\mu_{0}\right)\right\|$. The vector $\bar{\mu}_{0}=\boldsymbol{\Phi}^{T} \mu_{0}$ is the mean vector of all samples in the range space $V_{T}^{\perp}$. Take $\bar{\mu}_{0}$ as the spherical center, after all samples have been normalized, all of the samples in the range space are projected onto a unit hypersphere (Figure 1(a)). Then the eigenvectors of $\tilde{S}_{i}^{-1} \tilde{S}_{B i}$ can be calculated to extract the main features of the samples in class $i$. The unit vectors on the hypersphere not only show the proportion of each component of the original vectors, but also denote the direction. Thus, the range space hyperspherical discriminant analysis has good performance in pattern recognition.

The main different aspects of this paper by considering [27] relatively are as the following:(1)The main idea and final feature space between the two works are essentially different. RHDA maps data to a unit hypersphere and utilizes the Fisher criterion to calculate the eigenvectors of $\tilde{S}_{i}^{-1} \tilde{S}_{B i}$, then take these eigenvectors as projection matrix of the $i t h$ class samples . $\left(\tilde{S}_{i}\right.$ and $\tilde{S}_{B i}$, respectively, denote the within-class scatter matrix and between-class scatter matrix of class $i$ ). This shows RHDA is a study of subspace analysis approach and the final feature dimension of each sub-class is often different. RSCDA adds one dimension to every sample and maps it to a unit super spherical cap space. However, RSCDA does not calculate the projection matrix of each sub-class and the final feature dimensions of different sub-class which keep consistent.(2)The sample normalization process and intention are different. The unit hypersphere in $\mathrm{n}$-dimensional space is an (n-1)-dimensional manifold. Under the unit hypersphere model, the dimensions of transformed space are reduced from n-dimension to (n-1)-dimension, so the collinear sample points with spherical center would be overlapping. This would affect the design of classifiers and recognition accuracy. However, by adding one dimension to every sample, the unit super spherical cap model in $(n+1)$-dimensional space can keep the dimensions of transformed space unchanged, which can effectively overcome the sample fusion problem in the process of sample vectors normalization and keep sample separability. (3)The classifiers between two works are completely different. The classifier of RHDA is based on Euclidean distance, but this work proposes a new classifier called "spherical inner product nearest neighbor classifier" based on angle cosine distance. (4)The previous work utilizes a lot of experiments to verify the fact that the idea of sample vectors normalization has good performance and analyzes theoretically the optimal eigenvectors of each sub-class. The particular properties of the additional dimension, such as non-overlapping and separability, are analyzed theoretically by matrix theory in this work. The theoretical analyses are summarized as five lemmas in the section 3 .

\section{Range Space Unit Super Spherical Cap Mapping}

In the area of pattern classification, we expect that different classes maintain class separability after the mapping process and that the neighboring samples are still close to each other. However, there are two problems in the hypersphere mapping. One is the choice of spherical center. If there is a large difference of the sample number between different classes, the spherical center may be partial to the class that has many more samples. The other one is that the collinear sample points in the same direction will be overlapping under the hypersphere model. This means that the hypersphere mapping may cause a new separability problem. Based on the above analysis, we put forward a unit super spherical cap mapping model (Figure $1(\mathrm{~b}))$. 


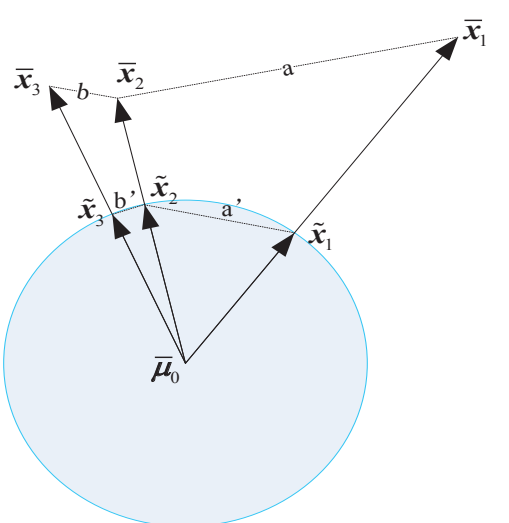

(a)

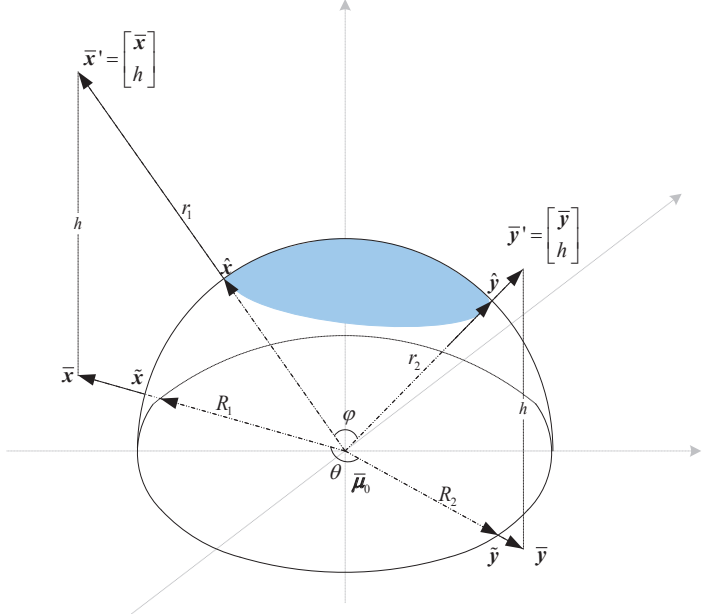

(b)

Figure 1: (a) Hyperspherical model $(a / b=4.9681)$; (b) Unit super spherical cap model.

\subsection{The unit super spherical cap mapping model}

Let $\overline{\mathbf{x}}=\left[\bar{x}_{1}, \bar{x}_{2}, \cdots, \bar{x}_{t_{T}}\right]^{T}$ be an arbitrary vector in the range space $V_{T}^{\perp}$. We add one component $h(0 \leq h<\infty)$ to it, and obtain a new vector $\overline{\mathbf{x}}^{\prime}=\left[\bar{x}_{1}, \bar{x}_{2}, \ldots, \bar{x}_{t_{T}}, h\right]$, where $\mathrm{h}$ is a real parameter. The unit super spherical cap mapping formula is described as follows

$$
\hat{\mathbf{x}}=\overline{\mathbf{x}}^{\prime} /\left\|\overline{\mathbf{x}}^{\prime}\right\|
$$

Then we have the following lemma 1.

Lemma 1. The relative proportion of each component of samples in the range space $V_{T}^{\perp}$ remains unchanged under the unit super spherical cap mapping model.

Proof. Let arbitrary vector $\overline{\mathbf{x}}=\left[\bar{x}_{1}, \bar{x}_{2}, \cdots, \bar{x}_{t_{T}}\right]^{T} \in V_{T}^{\perp}$, add one dimension to it and obtain a vector $\overline{\mathbf{x}}^{\prime}=\left[\bar{x}_{1}, \bar{x}_{2}, \cdots, \bar{x}_{t_{T}}, h\right]^{T}$. Corresponding, $\mathbf{a}=\left[\frac{\bar{x}_{1}^{2}}{\|\overline{\mathbf{x}}\|^{2}}, \cdots, \frac{\bar{x}_{t_{T}}^{2}}{\|\overline{\mathbf{x}}\|^{2}}\right]^{T}$ and $\mathbf{b}=\left[\frac{\bar{x}_{1}^{2}}{\|\overline{\mathbf{x}}\|^{2}+h^{2}}, \cdots, \frac{\bar{x}_{t_{T}}^{2}}{\|\overline{\mathbf{x}}\|^{2}+h^{2}}, \frac{h^{2}}{\|\overline{\mathbf{x}}\|^{2}+h^{2}}\right]^{T}$. Each element in a denotes the share of corresponding element in $\overline{\mathbf{x}}$ and the vector $\mathbf{b}$ has a similar meaning. Then, the relative proportion of arbitrary components $\bar{x}_{i}$ and $\bar{x}_{j}$ in $\overline{\mathbf{x}}$ can be calculated as $\frac{\bar{x}_{i}^{2}}{\|\overline{\mathbf{x}}\|^{2}} / \frac{\bar{x}_{j}^{2}}{\|\overline{\mathbf{x}}\|^{2}}=$ $\frac{\bar{x}_{i}^{2}}{\bar{x}_{j}^{2}}$. Similarly, the relative proportion of arbitrary components $\bar{x}_{i}$ and $\bar{x}_{j}$ in $\overline{\mathbf{x}}^{\prime}$ can be computed as $\frac{\bar{x}_{i}^{2}}{\|\overline{\mathbf{x}}\|^{2}+h^{2}} / \frac{\bar{x}_{j}^{2}}{\|\overline{\mathbf{x}}\|^{2}+h^{2}}=\frac{\bar{x}_{i}^{2}}{\bar{x}_{j}^{2}}$. That means the relative proportions of elements of samples are not changed after the unit super spherical cap mapping.

Lemma 2. Different samples in the range space $V_{T}^{\perp}$ will not overlap after the unit super spherical cap mapping.

Proof. Suppose $\overline{\mathbf{x}} \in V_{T}^{\perp}, \overline{\mathbf{y}} \in V_{T}^{\perp}$ and $\overline{\mathbf{x}} \neq \overline{\mathbf{y}}$. By equation (2), we can get $\hat{\mathbf{x}}=\overline{\mathbf{x}}^{\prime} /\left\|\overline{\mathbf{x}}^{\prime}\right\|$ and $\hat{\mathbf{y}}=\overline{\mathbf{y}}^{\prime} /\left\|\overline{\mathbf{y}}^{\prime}\right\|$, where $\overline{\mathbf{x}}^{\prime}=\left[\bar{x}_{1}, \bar{x}_{2}, \cdots, \bar{x}_{t_{T}}, h\right]^{T}$ and $\overline{\mathbf{y}}^{\prime}=\left[\bar{y}_{1}, \bar{y}_{2}, \cdots, \bar{y}_{t_{T}}, h\right]^{T}$. Our goal is to prove that $\hat{\mathbf{x}} \neq \hat{\mathbf{y}}$. The angle cosine between $\hat{\mathbf{x}}$ and $\hat{\mathbf{y}}$ can be computed as $\cos \theta=\hat{\mathbf{x}}^{T} \hat{\mathbf{y}}$, where $\hat{\mathbf{x}}^{T} \hat{\mathbf{y}}$ denotes the inner product between $\hat{\mathbf{x}}$ and $\hat{\mathbf{y}}$. Hence $\cos ^{2} \theta=\left(\hat{\mathbf{x}}^{T} \hat{\mathbf{y}}\right)^{2}=\left(\left(\overline{\mathbf{x}}^{\prime}\right)^{T} \overline{\mathbf{y}}^{\prime}\right)^{2} /\left(\left\|\overline{\mathbf{x}}^{\prime}\right\|^{2}\left\|\overline{\mathbf{y}}^{\prime}\right\|^{2}\right)$. We prove Lemma 2 by using the reduction to 
absurdity. Assuming that $\hat{\mathbf{x}}=\hat{\mathbf{y}}$, then $\cos ^{2} \theta=1$. Thus $\left\|\overline{\mathbf{x}}^{\prime}\right\|^{2}\left\|\overline{\mathbf{y}}^{\prime}\right\|^{2}-\left(\left(\overline{\mathbf{x}}^{\prime}\right)^{T} \overline{\mathbf{y}}^{\prime}\right)^{2}=0$. However,

$$
\begin{aligned}
& \left\|\overline{\mathbf{x}}^{\prime}\right\|^{2}\left\|\overline{\mathbf{y}}^{\prime}\right\|^{2}-\left(\left(\overline{\mathbf{x}}^{\prime}\right)^{T} \overline{\mathbf{y}}^{\prime}\right)^{2} \\
= & \left(\sum_{i=1}^{t_{T}} \bar{x}_{i}^{2}+h^{2}\right)\left(\sum_{i=1}^{t_{T}} \bar{y}_{i}^{2}+h^{2}\right)-\left(\sum_{i=1}^{t_{T}} \bar{x}_{i} \bar{y}_{i}+h^{2}\right)^{2} \\
= & {\left[\left(\sum_{i=1}^{t_{T}} \bar{x}_{i}^{2}\right)\left(\sum_{i=1}^{t_{T}} \bar{y}_{i}^{2}\right)+\left(\sum_{i=1}^{t_{T}} \bar{x}_{i}^{2}\right)\left(\sum_{i=1}^{t_{T}} \bar{y}_{i}^{2}\right) h^{2}+h^{4}\right]-\left[\left(\sum_{i=1}^{t_{T}} \bar{x}_{i} \bar{y}_{i}\right)^{2}+2 \sum_{i=1}^{t_{T}} \bar{x}_{i} \bar{y}_{i} h^{2}+h^{4}\right] } \\
= & \sum_{i=1}^{t_{T}} \bar{x}_{i}^{2} \bar{y}_{i}^{2}+\sum_{i=1}^{t_{T}} \sum_{j=1 ; j \neq i}^{t_{T}} \bar{x}_{i}^{2} \bar{y}_{j}^{2}+\sum_{i=1}^{t_{T}}\left(\bar{x}_{i}^{2}+\bar{y}_{i}^{2}\right) h^{2}-\left[\sum_{i=1}^{t_{T}} \bar{x}_{i}^{2} \bar{y}_{i}^{2}+\sum_{i=1}^{t_{T}-1} \sum_{j=i+1}^{t_{T}} 2 \bar{x}_{i} \bar{y}_{i} \bar{x}_{j} \bar{y}_{j}+\sum_{i=1}^{t_{T}} 2 \bar{x}_{i} \bar{y}_{i} h^{2}\right] \\
= & \sum_{i=1}^{t_{T}-1} \sum_{j=i+1}^{t_{T}}\left(\bar{x}_{i} \bar{y}_{j}-\bar{x}_{j} \bar{y}_{i}\right)^{2}+\sum_{i=1}^{t_{T}}\left(\bar{x}_{i}-\bar{y}_{i}\right)^{2} h^{2}
\end{aligned}
$$

Thus, we can obtain that $\bar{x}_{i}=\bar{y}_{i}\left(i=1,2, \cdots, t_{T}\right) \Rightarrow \overline{\mathbf{x}}=\overline{\mathbf{y}}$. This contradicts the known condition $\overline{\mathbf{x}} \neq \overline{\mathbf{y}}$. Thus, the assumption that $\hat{\mathbf{x}}=\hat{\mathbf{y}}$ is not correct and we obtain $\hat{\mathbf{x}} \neq \hat{\mathbf{y}}$.

The collinear vectors that have different lengths would be overlapped after the hypersphere mapping. However, under the same condition, Lemma 2 shows that the overlapping phenomenon would not occur under the unit super spherical cap mapping model.

\subsection{The distance relation of sample vectors on the unit super spherical cap}

Lemma 3. Suppose $\hat{\mathbf{x}}$ and $\hat{\mathbf{y}}$ are two different unit vectors on the unit super spherical cap, then we have the follow equations

$$
d^{2}(\hat{\mathbf{x}}-\hat{\mathbf{y}})=2(1-\cos \theta)=2\left(1-\hat{\mathbf{x}}^{T} \hat{\mathbf{y}}\right)
$$

where $\theta \in[0, \pi]$ and $d(\hat{\mathbf{x}}-\hat{\mathbf{y}})$ denote the angle and the Euclidean distance between the vectors $\hat{\mathbf{x}}$ and $\hat{\mathbf{y}}$, respectively.

Proof. Because $\hat{\mathbf{x}}$ and $\hat{\mathbf{y}}$ are both unit vectors, $d(\hat{\mathbf{x}}-\hat{\mathbf{y}})$ and $\theta$ satisfy the relation $d^{2}(\hat{\mathbf{x}}, \hat{\mathbf{y}})=(\sin \theta)^{2}+$ $(1-\cos \theta)^{2}(\operatorname{Fig} 2(\mathrm{a})(\mathrm{b}))$, which implies $d^{2}(\hat{\mathbf{x}}, \hat{\mathbf{y}})=2(1-\cos \theta)$. Alternately, the inner product of $\hat{\mathbf{x}}$ and $\hat{\mathbf{y}}$ can be calculated as $\hat{\mathbf{x}}^{T} \hat{\mathbf{y}}=\cos \theta$. Thus, we obtain the equations (3). The functional relation between $d(\hat{\mathbf{x}}-\hat{\mathbf{y}})$ and $\theta$ as shown in Figure $2(\mathrm{c})$.

Let arbitrary vectors $\overline{\mathbf{x}}=\left[\bar{x}_{1}, \bar{x}_{2}, \cdots, \bar{x}_{t_{T}}\right]^{T} \in V_{T}^{\perp}, \overline{\mathbf{y}}=\left[\bar{y}_{1}, \bar{y}_{2}, \cdots, \bar{y}_{t_{T}}\right]^{T} \in V_{T}^{\perp}, \overline{\mathbf{x}}^{\prime}=\left[\bar{x}_{1}, \bar{x}_{2}, \cdots, \bar{x}_{t_{T}}, h\right]^{T}$ and $\overline{\mathbf{y}}^{\prime}=\left[\bar{y}_{1}, \bar{y}_{2}, \cdots, \bar{y}_{t_{T}}, h\right]^{T}$. Then it is easy to obtain $\hat{\mathbf{x}}$ and $\hat{\mathbf{y}}$ by the equation (2). Denote $R_{1}=\sqrt{\sum_{i=1}^{t_{T}} \bar{x}_{i}^{2}}=$ $\|\overline{\mathbf{x}}\|, R_{2}=\sqrt{\sum_{i=1}^{t_{T}} \bar{y}_{i}^{2}}=\|\overline{\mathbf{y}}\|, r_{1}=\sqrt{R_{1}^{2}+h^{2}}=\left\|\overline{\mathbf{x}}^{\prime}\right\|, r_{2}=\sqrt{R_{2}^{2}+h^{2}}=\left\|\overline{\mathbf{y}}^{\prime}\right\|$, we then have the following conclusion.

Lemma 4. $d(\overline{\mathbf{x}}, \overline{\mathbf{y}})$ and $d(\hat{\mathbf{x}}, \hat{\mathbf{y}})$ satisfy the following equation

$$
d^{2}(\hat{\mathbf{x}}, \hat{\mathbf{y}})=\frac{d^{2}(\overline{\mathbf{x}}, \overline{\mathbf{y}})}{r_{1} r_{2}}-\left(\frac{r_{1}}{r_{2}}+\frac{r_{2}}{r_{1}}\right)+2,
$$

where $d(\overline{\mathbf{x}}, \overline{\mathbf{y}})$ denotes the Euclidean distance between $\overline{\mathbf{x}}$ and $\overline{\mathbf{y}}$, similarly to $d(\hat{\mathbf{x}}, \hat{\mathbf{y}})$. 


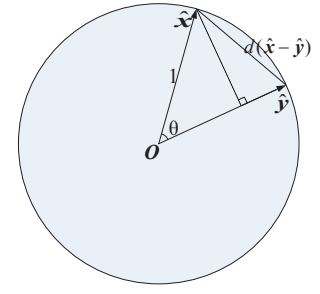

(a)

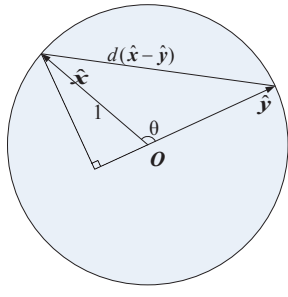

(b)

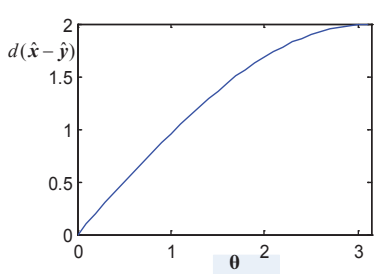

(c)

Figure 2: (a) $\theta \in(0, \pi / 2)$; (b) $\theta \in[\pi / 2, \pi)$; (c)the function relationship between $\theta$ and $d(\hat{\mathbf{x}}-\hat{\mathbf{y}})$

Proof. According to the theory of functional analysis, one has

$$
d^{2}(\overline{\mathbf{x}}, \overline{\mathbf{y}})=(\overline{\mathbf{x}}-\overline{\mathbf{y}})^{T}(\overline{\mathbf{x}}-\overline{\mathbf{y}})=R_{1}^{2}+R_{2}^{2}-2 \sum_{i=1}^{t_{T}} \bar{x}_{i} \bar{y}_{i}
$$

By Lemma 3 and the equation (3),

$$
d^{2}(\hat{\mathbf{x}}, \hat{\mathbf{y}})=2-2 \hat{\mathbf{x}}^{T} \hat{\mathbf{y}}=2-\frac{2\left(\overline{\mathbf{x}}^{\prime}\right)^{T} \overline{\mathbf{y}}^{\prime}}{\left\|\overline{\mathbf{x}}^{\prime}\right\|\left\|\overline{\mathbf{y}}^{\prime}\right\|}=2-\left(2 \sum_{i=1}^{t_{T}} \bar{x}_{i} \bar{y}_{i}+2 h^{2}\right) /\left(r_{1} r_{2}\right)
$$

Combining Eqs (5) and (6), we obtain

$$
d^{2}(\hat{\mathbf{x}}, \hat{\mathbf{y}})=\frac{d^{2}(\overline{\mathbf{x}}, \overline{\mathbf{y}})}{r_{1} r_{2}}-\left(\frac{r_{1}}{r_{2}}+\frac{r_{2}}{r_{1}}\right)+2
$$

Lemma 4 shows that, for the given samples $\overline{\mathbf{x}}$ and $\overline{\mathbf{y}}$, when parameter $h$ has been selected, the distance $d(\hat{\mathbf{x}}, \hat{\mathbf{y}})$ will increase with the distance $d(\overline{\mathbf{x}}, \overline{\mathbf{y}})$. In particular, when $\overline{\mathbf{x}}$ and $\overline{\mathbf{y}}$ have the same length, namely, $r_{1}=$ $r_{2}$, the original distance $d(\overline{\mathbf{x}}, \overline{\mathbf{y}})$ is directly proportional to the mapped distance $d(\hat{\mathbf{x}}, \hat{\mathbf{y}})$. This result shows that the super spherical cap mapping model can maintain the topological invariance of the distance between samples. Under the super spherical cap model, the degree of aggregation of mapped samples is associated with the parameter $h$. The larger $h$ we chose, the more concentrated the degree of aggregation would be. If $h=0$, the super spherical cap mapping model degenerates to the hypersphere mapping model.

\subsection{The separability of the super spherical cap model is based on modified FLDA criterion}

In this subsection, to simplify the problem, we only consider the database consist of two classes, where the $i$ th class contains $N_{i}$ samples. Thus, there are a total of $N=\sum_{i=1}^{2} N_{i}$ samples. In the range space $V_{T}^{\perp}$, let $\overline{\mathbf{x}}_{i}(i=1,2 \ldots N)$ denotes the $i$ th feature vector, $\bar{\mu}_{k}(k=1,2)$ is the mean of samples in the $k$ th class and $\bar{\mu}_{0}$ is the mean of all samples. Suppose that $\bar{\mu}_{0}=0$, Otherwise, we take each sample subtract the mean $\bar{\mu}_{0}$ and obtain a new sample vector set. Obviously, the total mean of the new sample set equals zero-mean. Applying formula (2) to obtain a new vector $\hat{\mathbf{x}}_{i}=\left(\overline{\mathbf{x}}_{i}^{T}, h\right)^{T} / \sqrt{\left\|\overline{\mathbf{x}}_{i}\right\|^{2}+h^{2}}$, it denotes a unit vector on the unit super spherical cap manifold. Accordingly, after unit super spherical cap mapping, $\hat{\mu}_{k}(k=1,2)$ denotes the mean of samples in the $k$ th class, and $\hat{\mu}_{0}$ denotes the total mean of all samples. Then, we have the following conclusion.

Lemma 5. If $\overline{\mathbf{x}}_{i} \in V_{T}^{\perp}$ satisfies the condition $\frac{1}{N} \sum_{i=1}^{N}\left\|\overline{\mathbf{x}}_{i}\right\|^{2}>2\left\|\bar{\mu}_{1}-\bar{\mu}_{2}\right\|^{2}\left(1-\left\|\hat{\mu}_{0}\right\|^{2}\right) /\left\|\hat{\mu}_{1}-\hat{\mu}_{2}\right\|^{2}$, then the unit super spherical cap mapping model will improve the separability of samples. 
Proof. Let $\overline{\mathbf{S}}_{B}$ and $\overline{\mathbf{S}}_{T}$, respectively, denote the between-class scatter matrix and total scatter matrix of samples in the range space $V_{T}^{\perp}$. Similarly, $\hat{\mathbf{S}}_{B}$ and $\hat{\mathbf{S}}_{T}$ are the between-class scatter matrix and total scatter matrix of samples on the unit super spherical cap manifold. When the database is composed of two classes, it is easy to prove that $\hat{\mathbf{S}}_{B}=\sum_{i=1}^{2} N_{i}\left(\hat{\mu}_{i}-\hat{\mu}_{0}\right)\left(\hat{\mu}_{i}-\hat{\mu}_{0}\right)^{T}=\left(\hat{\mu}_{1}-\hat{\mu}_{2}\right)\left(\hat{\mu}_{1}-\hat{\mu}_{2}\right)^{T} N_{1} N_{2} / N . \overline{\mathbf{S}}_{B}$ has a similar expression. Let $\operatorname{tr}(\bullet)$ denotes the trace of a matrix, using matrix theory, we have

$$
\begin{aligned}
\operatorname{tr}\left(\hat{\mathbf{S}}_{B}\right) \operatorname{tr}\left(\overline{\mathbf{S}}_{T}\right) & =\operatorname{tr}\left[\left(\hat{\mu}_{1}-\hat{\mu}_{2}\right)\left(\hat{\mu}_{1}-\hat{\mu}_{2}\right)^{T} \frac{N_{1} N_{2}}{N}\right] \operatorname{tr}\left[\sum_{i=1}^{N}\left(\overline{\mathbf{x}}_{i}-\bar{\mu}_{0}\right)\left(\overline{\mathbf{x}}_{i}-\bar{\mu}_{0}\right)^{T}\right] \\
& =\left(\hat{\mu}_{1}-\hat{\mu}_{2}\right)^{T}\left(\hat{\mu}_{1}-\hat{\mu}_{2}\right) \frac{N_{1} N_{2}}{N} \sum_{i=1}^{N} \operatorname{tr}\left(\overline{\mathbf{x}}_{i} \overline{\mathbf{x}}_{i}^{T}\right)=\frac{N_{1} N_{2}}{N}\left\|\hat{\mu}_{1}-\hat{\mu}_{2}\right\|^{2} \sum_{i=1}^{N}\left\|\overline{\mathbf{x}}_{i}\right\|^{2}
\end{aligned}
$$

On the other hand, we have

$$
\begin{aligned}
& \operatorname{tr}\left(\hat{\mathbf{S}}_{T}\right) \operatorname{tr}\left(\overline{\mathbf{S}}_{B}\right) \\
& =\operatorname{tr}\left\{\sum_{i=1}^{N}\left[\left(\hat{\mathbf{x}}_{i}-\hat{\mu}_{0}\right)\left(\hat{\mathbf{x}}_{i}-\hat{\mu}_{0}\right)^{T}\right]\right\} \operatorname{tr}\left[\left(\bar{\mu}_{1}-\bar{\mu}_{2}\right)\left(\bar{\mu}_{1}-\bar{\mu}_{2}\right)^{T} \frac{N_{1} N_{2}}{N}\right] \\
& =\sum_{i=1}^{N} \operatorname{tr}\left[\left(\hat{\mathbf{x}}_{i}-\hat{\mu}_{0}\right)\left(\hat{\mathbf{x}}_{i}-\hat{\mu}_{0}\right)^{T}\right]\left\|\bar{\mu}_{1}-\bar{\mu}_{2}\right\|^{2} \frac{N_{1} N_{2}}{N} \\
& =\frac{N_{1} N_{2}}{N} \sum_{i=1}^{N}\left\|\hat{\mathbf{x}}_{i}-\hat{\mu}_{0}\right\|^{2}\left\|\bar{\mu}_{1}-\bar{\mu}_{2}\right\|^{2} \\
& =\frac{N_{1} N_{2}}{N} \sum_{i=1}^{N}\left\|\frac{\left(\overline{\mathbf{x}}_{i}^{T}, h\right)^{T}}{\sqrt{\left\|\overline{\mathbf{x}}_{i}\right\|^{2}+h^{2}}}-\frac{1}{N} \sum_{k=1}^{N} \frac{\left(\overline{\mathbf{x}}_{k}^{T}, h\right)^{T}}{\sqrt{\left\|\overline{\mathbf{x}}_{k}\right\|^{2}+h^{2}}}\right\|^{2}\left\|\bar{\mu}_{1}-\bar{\mu}_{2}\right\|^{2} \\
& =\frac{N_{1} N_{2}}{N} \sum_{i=1}^{N}\left[\frac{\left(\overline{\mathbf{x}}_{i}^{T}, h\right)}{\sqrt{\left\|\overline{\mathbf{x}}_{i}\right\|^{2}+h^{2}}}-\frac{1}{N} \sum_{k=1}^{N} \frac{\left(\overline{\mathbf{x}}_{k}^{T}, h\right)}{\sqrt{\left\|\overline{\mathbf{x}}_{k}\right\|^{2}+h^{2}}}\right]\left[\frac{\left(\overline{\mathbf{x}}_{i}^{T}, h\right)^{T}}{\sqrt{\left\|\overline{\mathbf{x}}_{i}\right\|^{2}+h^{2}}}-\frac{1}{N} \sum_{k=1}^{N} \frac{\left(\overline{\mathbf{x}}_{k}^{T}, h\right)^{T}}{\sqrt{\left\|\overline{\mathbf{x}}_{k}\right\|^{2}+h^{2}}}\right]\left\|\bar{\mu}_{1}-\bar{\mu}_{2}\right\|^{2} \\
& \leq \frac{N_{1} N_{2}}{N}\left\{\sum_{i=1}^{N} \frac{\left(\overline{\mathbf{x}}_{i}^{T}, h\right)\left(\overline{\mathbf{x}}_{i}^{T}, h\right)^{T}}{\left\|\overline{\mathbf{x}}_{i}\right\|^{2}+h^{2}}-\frac{2}{N} \sum_{k=1}^{N} \frac{\left(\overline{\mathbf{x}}_{k}^{T}, h\right)}{\sqrt{\left\|\overline{\mathbf{x}}_{k}\right\|^{2}+h^{2}}} \sum_{i=1}^{N} \frac{\left(\overline{\mathbf{x}}_{i}^{T}, h\right)^{T}}{\sqrt{\left\|\overline{\mathbf{x}}_{i}\right\|^{2}+h^{2}}}+N\right\}\left\|\bar{\mu}_{1}-\bar{\mu}_{2}\right\|^{2} \\
& =\frac{N_{1} N_{2}}{N}\left\{2 N-2 N\left[\frac{1}{N} \sum_{k=1}^{N} \frac{\left(\overline{\mathbf{x}}_{k}^{T}, h\right)}{\sqrt{\left\|\overline{\mathbf{x}}_{k}\right\|^{2}+h^{2}}}\right]\left[\frac{1}{N} \sum_{i=1}^{N} \frac{\left(\overline{\mathbf{x}}_{i}^{T}, h\right)^{T}}{\sqrt{\left\|\overline{\mathbf{x}}_{i}\right\|^{2}+h^{2}}}\right]\right\}\left\|\bar{\mu}_{1}-\bar{\mu}_{2}\right\|^{2} \\
& =2 N_{1} N_{2}\left\{1-\left\|\frac{1}{N} \sum_{k=1}^{N} \frac{\left(\overline{\mathbf{x}}_{k}^{T}, h\right)}{\sqrt{\left\|\overline{\mathbf{x}}_{k}\right\|^{2}+h^{2}}}\right\|^{2}\right\}\left\|\bar{\mu}_{1}-\bar{\mu}_{2}\right\|^{2}=2 N_{1} N_{2}\left(1-\left\|\hat{\mu}_{0}\right\|^{2}\right)\left\|\bar{\mu}_{1}-\bar{\mu}_{2}\right\|^{2}
\end{aligned}
$$

Considering that $\overline{\mathbf{x}}_{i}$ satisfies the condition $\frac{1}{N} \sum_{i=1}^{N}\left\|\overline{\mathbf{x}}_{i}\right\|^{2}>2\left\|\bar{\mu}_{1}-\bar{\mu}_{2}\right\|^{2}\left(1-\left\|\hat{\mu}_{0}\right\|^{2}\right) /\left\|\hat{\mu}_{1}-\hat{\mu}_{2}\right\|^{2}$, we have

$$
\frac{N_{1} N_{2}}{N}\left\|\hat{\mu}_{1}-\hat{\mu}_{2}\right\|^{2} \cdot \sum_{i=1}^{N}\left\|\overline{\mathbf{x}}_{i}\right\|^{2}>2 N_{1} N_{2}\left(1-\left\|\hat{\mu}_{0}\right\|^{2}\right)\left\|\bar{\mu}_{1}-\bar{\mu}_{2}\right\|^{2}
$$

Combining equation (8),inequalities (9) and (10), we obtain

$$
\frac{\operatorname{tr}\left(\hat{\mathbf{S}}_{B}\right)}{\operatorname{tr}\left(\hat{\mathbf{S}}_{T}\right)}>\frac{\operatorname{tr}\left(\overline{\mathbf{S}}_{B}\right)}{\operatorname{tr}\left(\overline{\mathbf{S}}_{T}\right)}
$$


As shown in (11), we use modified Fisher criterion function $F(q)=q^{T} \mathbf{S}_{B} q / q^{T} \mathbf{S}_{T} q$ [29]-[30] to build separability criterion. Lemma 5 shows that, when the average of the square length of all of the samples is larger than $K$ times the square distance of two class means, the unit super spherical cap mapping model can improve the separability of samples. Here $K=2\left(1-\left\|\hat{\mu}_{0}\right\|^{2}\right) /\left\|\hat{\mu}_{1}-\hat{\mu}_{2}\right\|^{2}$. In fact, for the given sample data, we can calculate $\hat{\mu}_{1}, \hat{\mu}_{2}, \hat{\mu}_{0}$ and $K$. The inequality (11) provides a quantitative criterion for comparing the separability of the data set. Generally speaking, the size of $K$ becomes large, the possibility that the condition of Lemma 5 is satisfied becomes small. The size of $K$ reflects the separability of mapped data to some extent. For the sample vectors which projected onto the super spherical cap model, when the mean center $\hat{\mu}_{0}$ is selected, the smaller $K$ is, the larger $\left\|\hat{\mu}_{1}-\hat{\mu}_{2}\right\|$ is. That means the between-class distance becomes larger, which may improve the separability of mapped data.

\section{A new classifier based on the angle cosine distance in the unit super spherical cap manifold $C_{V_{T}^{\perp}}$}

In this section, we propose a new classifier called the "spherical inner product nearest neighbor classifier" (Fig 3) for the data in $C_{V_{T}^{\perp}}$. For each sample $\hat{\mathbf{x}}_{k}^{i}\left(k=1,2, \ldots, N_{i}\right)$ in the $i$ th class, the nearest sample to $\hat{\mathbf{x}}_{k}^{i}\left(k=1,2, \ldots, N_{i}\right)$, that doesn't belong to the $i$ th class is especially noteworthy. These samples at the boundary decide the region of class $i$.

Let the arbitrary vector $\hat{\mathbf{x}}_{k}^{i}$ be in the $i$ th class, and $\hat{\mathbf{x}}_{j}^{p}$ not be in the $i$ th class,

$$
\theta_{k}^{i}=\max _{\theta_{j}} \arg \left(\cos \theta_{j}=\frac{\left(\hat{\mathbf{x}}_{k}^{i}\right)^{T} \hat{\mathbf{x}}_{j}^{p}}{\left\|\hat{\mathbf{x}}_{k}^{i}\right\|\left\|\hat{\mathbf{x}}_{j}^{p}\right\|}\right)
$$

where $i=1, \cdots, C ; k=1, \cdots, N_{i} ; j \in\left\{1, \cdots, N_{p}\right\} ; p \in\{1, \cdots, i-1, i+1, \ldots C\}$ and $\left\|\hat{\mathbf{x}}_{k}^{i}\right\|=\left\|\hat{\mathbf{x}}_{j}^{p}\right\|=1$. Let $t_{k}^{i}=\theta_{k}^{i} / 2$, RSCDA classifies test samples into class $i$, but only the test samples located in the internal of the small circles $O\left(\hat{\mathbf{x}}_{k}^{i}, t_{k}^{i}\right)$, which take a certain vector of class $i$ as the circle center with a spherical radius of $t_{k}^{i}$. Because there are $N_{i}$ samples in the $i$ th class, we can obtain $N_{i}$ circles that decide the region of class $i$.

For a new test sample $\hat{\mathbf{x}}$, if a training sample $\hat{\mathbf{x}}_{k}^{i}\left(i=1,2, \cdots, C ; k=1,2, \cdots, N_{i}\right)$, exsits such that $\hat{\mathbf{x}}^{T} \hat{\mathbf{x}}_{k}^{i} \geq \cos \left(t_{k}^{i}\right)$, then $\hat{\mathbf{x}}$ belongs to class $i$. Otherwise, we consider $\hat{\mathbf{x}}$ to be a new pattern class.

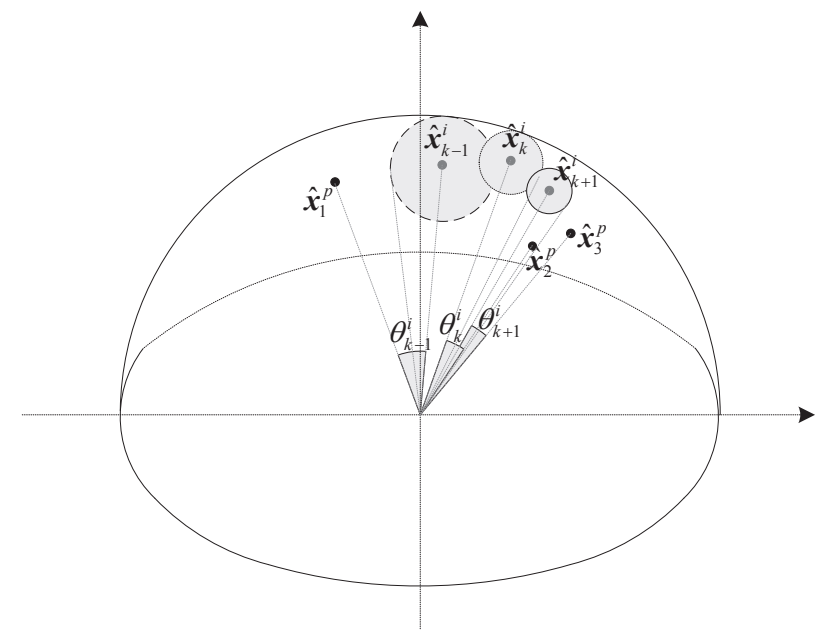

Figure 3: Spherical inner product nearest neighbor classifier. 


\section{The incremental learning of the proposed classifier and algorithm steps}

In recent years, incremental learning has been a very interesting research direction in pattern recognition. Based on the proposed model and classifier, it is natural to propose an incremental learning method. If a new training sample $\hat{\mathbf{x}}_{n e w}^{i}$ is added in the $i$ th class, we can use formula (12) to calculate a new angle $\theta_{n e w}^{i}$. Thus, we obtain a new threshold value $t_{\text {new }}^{i}=\theta_{\text {new }}^{i} / 2$ and a new circle $o\left(\hat{\mathbf{x}}_{\text {new }}^{i}, t_{\text {new }}^{i}\right)$. We add the circle to the $i$ th class classifier to update the region of class $i$.

The range space super spherical cap discriminant analysis algorithm (RSCDA) can be summarized as follows.

Step 1. Calculate mean vectors $\mu_{0}, \mu_{1}, \cdots, \mu_{C}$ and total scatter matrix $\mathbf{S}_{T}$ according to formula (1).

Step 2. Calculate the eigenvectors $\phi_{1} \phi_{2} \cdots \phi_{t_{T}}$ corresponding to nonzero eigenvalues of $\mathbf{S}_{T}$ using the algorithm in [12] and obtain the range space $V_{T}^{\perp}$, where $V_{T}^{\perp}=\operatorname{span}\left(\phi_{1}, \cdots, \phi_{t_{T}}\right)$ and $t_{T}=\operatorname{Rank}\left(\mathbf{S}_{T}\right)$.

Step 3. Project the training samples to the surface of the unit super spherical cap manifold. Let $\mathbf{x}_{k}^{i}$ denote an original sample and $\hat{\mathbf{x}}_{k}^{i}$ denote a projected sample. Compute $\hat{\mathbf{x}}_{k}^{i}$ by the formula $\hat{\mathbf{x}}_{k}^{i}=\left(\overline{\mathbf{x}}_{k}^{i}\right)^{\prime} /\left\|\left(\overline{\mathbf{x}}_{k}^{i}\right)^{\prime}\right\|$, where $\left(\overline{\mathbf{x}}_{k}^{i}\right)^{\prime}=\left[\begin{array}{c}\overline{\mathbf{x}}_{k}^{i} \\ h\end{array}\right], \overline{\mathbf{x}}_{k}^{i}=\boldsymbol{\Phi}^{T}\left(\mathbf{x}_{k}^{i}-\mu_{0}\right)$ and $\boldsymbol{\Phi}=\left[\phi_{1}, \cdots, \phi_{t_{T}}\right]\left(i=1, \cdots, C ; k=1, \cdots, N_{i}\right)$.

Step 4. Calculate the threshold value $t_{k}^{i}=\theta_{k}^{i} / 2$ for each class, where $\theta_{k}^{i}$ can be obtained by equation (12). Step 5. Map the test sample $\mathbf{x}$ to $\hat{\mathbf{x}}$ by using the method of step 3 .

Step 6. Compute the inner product between $\hat{\mathbf{x}}$ and each training sample $\hat{\mathbf{x}}_{k}^{i}$. If a training sample $\hat{\mathbf{x}}_{k}^{i}$ exists such that $\hat{\mathbf{x}}^{T} \hat{\mathbf{x}}_{k}^{i} \geq \cos \left(t_{k}^{i}\right)$, then $\hat{\mathbf{x}}$ belongs to the $i$ th class. Otherwise, we consider $\hat{\mathbf{x}}$ to be a new pattern class.

Step 7. If there is a new training sample, we could apply the incremental learning method to update all the regions of the super spherical cap.

The space transformation process of RSCDA is shown in Figure 4.

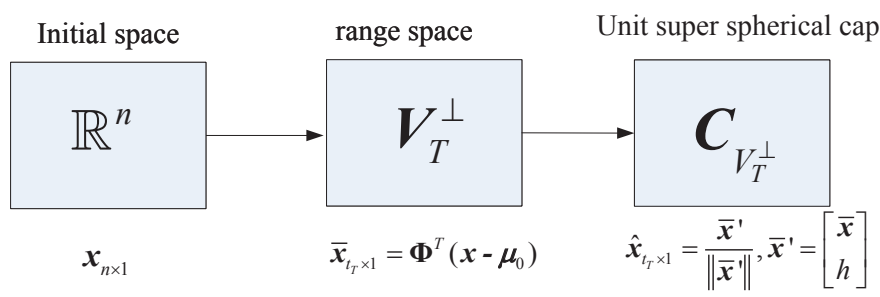

Figure 4: Space transformation process of RSCDA.

\section{Experimental results}

To effectively reduce the loss of discriminant information, OFLD, FDNS, FFS and OSNS extract different types of feature vectors in the range space or null space. We compared the performance of RSCDA with that of FLD, OFLD,PNBDA, FDNS, OSNS, SK-PCA and FFS. The Image Segmentation, Iris, Double-moon, Caltech-101 and COIL100 databaes were used to test these algorithms. The Nearest Neighbor Co-sharing level Orientation (NNCO) plot of some databases show the sample characteristics of different classes in different low-dimensional subspaces or manifolds [31]. The proposed method adopted the classifier as shown in Figure 3, and the NN classifier was employed for the others. The training samples were selected randomly in each run, while the remaining were used for testing. The result was the average of five runs.

It is important to select the appropriate parameter $h$ for our algorithm. If $h$ is too large, the degree of aggregation of the sample vectors would be very high and the weight of other components in $\overline{\mathbf{x}}^{\prime}$ would become very small, which will affect the recognition accuracy of our method. Conversely, if $h$ is too small, the overlapping problem in the process of sample vectors normalization may not be fully resolved. Our parameter selection strategy based on the idea that keeps $h$ and the average length in all dimensions of 
Table 1: Classification error probability for the Image Segmentation database.

\begin{tabular}{lccc}
\hline Algorithms & Minimum error & Maximum error & Mean error \\
\hline FLDA & 0.1348 & 0.2690 & 0.1978 \\
OFLD & 0.1168 & 0.1445 & 0.1298 \\
PNBDA & 0.0585 & 0.0740 & 0.0676 \\
FDNS & 0.1623 & 0.2294 & 0.1876 \\
OSNS & 0.0590 & 0.0735 & 0.0685 \\
SK-PCA & 0.1426 & 0.2033 & 0.1624 \\
FFS & 0.0768 & 0.1430 & 0.0946 \\
RSCDA & 0.0585 & 0.0710 & 0.0625 \\
\hline
\end{tabular}

Table 2: The average total CPU time (s) for each method on the Image Segmentation database.

\begin{tabular}{lcccccccc}
\hline Algorithms & FLDA & OFLD & PNBDA & FDNS & OSNS & SK-PCA & FFS & RSCDA \\
\hline Running time & 16.57 & 221.50 & 290.28 & 278.66 & 389.53 & 38.55 & 129.40 & 395.66 \\
\hline
\end{tabular}

samples in the same order of magnitude. The adjustable parameter is $h=r * d$, where $d$ denotes the average length in all dimensions of the samples in the range space $V_{T}^{\perp}$ and $r$ is an adjustable positive constant. In the subsequent experiments, $r$ varies from 0 to 100 with a step of 0.5 and we select the appropriate parameter $r$ to obtain satisfactory recognition accuracy.

6.1 Experiments with the Image Segmentation database

The Image Segmentation database has 2310 samples from seven outdoor images: grass, path, window, cement, foliage, sky, and brickface. There are 330 samples in each class and each sample corresponds to a region with 19 attributes. These attributes are region-centroid-col, region-centroid-row, region-pixelcount, short-line-density-5, short-line-density-2, vedge-mean, vegde-sd, hedge-mean, hedge-sd, intensitymean, rawred-mean, rawblue-mean, rawgreen-mean, exred-mean, exblue-mean, exgreen-mean, value-mean, saturation-mean and hue-mean.

100 samples from each class were randomly chosen for the experiment. The parameter $r$ of our method was chosen as 10. The maximum error and the mean error of RSCDA are the lowest among the eight compared algorithms (Table1). FLDA is suitable for the same covariance matrix between different classes, but RSCDA is not restricted by this condition. OFLD, FDNS, OSNS, SK-PCA and FFS can effectively extract various types of discriminant information based on Euclidean distance information and obtain good recognition rates. However, for data that have different low-dimensional structure, both distance and angle information should be considered. Moreover, the proposed method does not lose effective discriminant information in the process of range space transformation. These are the main reasons why RSCDA outperforms the others.

Furthermore, 120 samples from each class were randomly chosen for training and the others were used for testing. The average total CPU time (s) for each method is listed in Table 2.

In fact, "How does $r$ influence RSCDA" is an interesting question. Randomly selected 120 samples for training, and varied the parameter $r$ from 0 to 100 with an interval of 0.5 . From the blue curve in the Fig.5(a), we can see that when $r=0$, the recognition of RSCDA is the lowest. The main reason is that, the super spherical cap degenerates to the hypersphere model, which leads to overlapping in the process of samples unitization. The parameter $r$ in our method is unique and the parameters of other methods have different forms. By adjusting the parameters of the remainder methods, the optimal mean recognition rates of related works are represented by the horizontal lines in the Fig.5(a). More specifically, we use corresponding numerical recognition rates to denote all the horizontal lines as well as the average of the RSCDA curve value $(10<r<100)$ in Table 3. Moreover, As shown in the Fig.5(b), when $\log _{e} r>17$, the average recognition rate of our approach will tend to drop off. This means that our approach has good robustness for different parameter $r$ within a certain range.

6.2 Experiments with the Iris database

The Iris database contains 150 samples from three classes: Iris-setosa, Iris-versicolor, and Iris-virginica, with 
Table 3: Recognition rate for the Image Segmentation database.

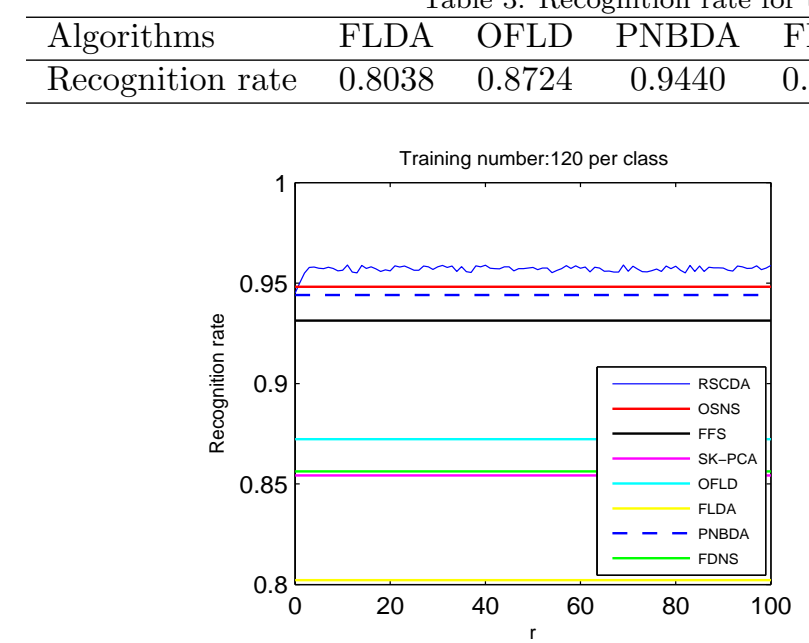

(a)

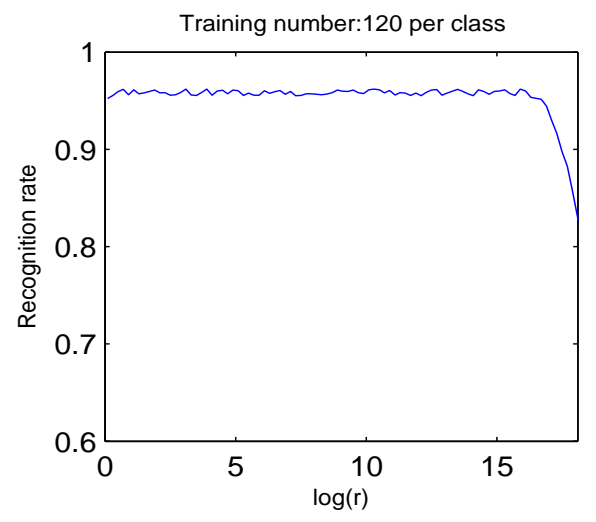

(b)

Figure 5: Recognition rate for the Image Segmentation database . (a) $0<r<100$; (b) $0<\log _{e}(r)<18$.

50 samples each. Each sample has four numeric attributes: sepal length, sepal width, petal length, and petal width. As shown in [31],the first class is linearly separable from the other two classes, and the latter two are not linearly separable from each other. 22 samples were selected randomly from each class for training and the parameter of RSCDA was $r=5$. As we can see clearly from Table 4, OSNS and RSCDA have the same minimum error, and the proposed approach has the lowest recognition maximum error and mean error. In addition, randomly selected 25 samples for training and the rest for testing. The average total CPU time ( $\mathrm{s}$ ) for each method is listed in Table 5, and the influence of parameter $r$ for RSCDA and optimal recognition rates of related methods are showed in Fig. 6 and Table 6.

\subsection{Experiments with the Double-moon database}

This experiment was performed on the Double-moon database from "Neural Networks and learning Machines" written by Simon Haykin [32]. As shown in Fig. 7(a), the Double-moon database consists of two asymmetric moons. The radius of each moon is 5, and the vertical distance between the two moons is -3 . The red moon represents the first class sample point and the blue moon denotes another class. There are 1000 samples in each class and each sample is two-dimensional.

The parameter is $r=6$. By calculation, $\frac{1}{N} \sum_{i=1}^{N}\left\|\overline{\mathbf{x}}_{i}\right\|^{2}=32.9568,\left\|\bar{\mu}_{1}-\bar{\mu}_{2}\right\|=6.0425$ and $K=0.88$. It can be easily verified that such parameters meet the condition of Lemma 5 . Moreover, we find that $0.2770=\operatorname{tr}\left(\overline{\mathbf{S}}_{B}\right) / \operatorname{tr}\left(\overline{\mathbf{S}}_{T}\right)<\operatorname{tr}\left(\hat{\mathbf{S}}_{B}\right) / \operatorname{tr}\left(\hat{\mathbf{S}}_{T}\right)=0.3558$. This means the proposed model can effectively improve

Table 4: Classification error probability for the Iris database.

\begin{tabular}{lccc}
\hline Algorithms & Minimum error & Maximum error & Mean error \\
\hline FLDA & 0.0400 & 0.0800 & 0.0511 \\
OFLD & 0.0400 & 0.1000 & 0.0600 \\
PNBDA & 0.0267 & 0.0667 & 0.0489 \\
FDNS & 0.0267 & 0.0533 & 0.0467 \\
OSNS & 0.0133 & 0.0567 & 0.0415 \\
SK-PCA & 0.0246 & 0.0800 & 0.0565 \\
FFS & 0.0246 & 0.0567 & 0.0425 \\
RSCDA & 0.0133 & 0.0533 & 0.0347 \\
\hline
\end{tabular}


Table 5: The average total CPU time (s) for each method on the Iris database.

\begin{tabular}{lcccccccc}
\hline Algorithms & FLDA & OFLD & PNBDA & FDNS & OSNS & SK-PCA & FFS & RSCDA \\
\hline Running time & 0.80 & 1.06 & 1.40 & 1.36 & 1.54 & 1.05 & 1.18 & 1.55 \\
\hline
\end{tabular}

Table 6: Recognition rate for the Iris database.

\begin{tabular}{lcccccccc}
\hline Algorithms & FLDA & OFLD & PNBDA & FDNS & OSNS & SK-PCA & FFS & RSCDA(average) \\
\hline Recognition rate & 0.9539 & 0.9450 & 0.9561 & 0.9583 & 0.9640 & 0.9485 & 0.9615 & 0.9680 \\
\hline
\end{tabular}

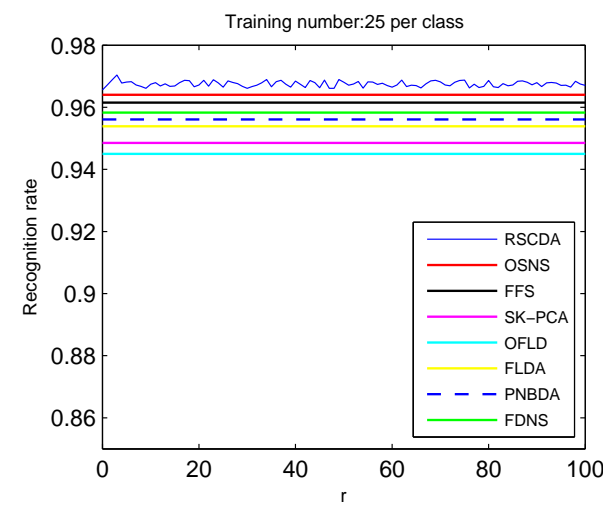

(a)

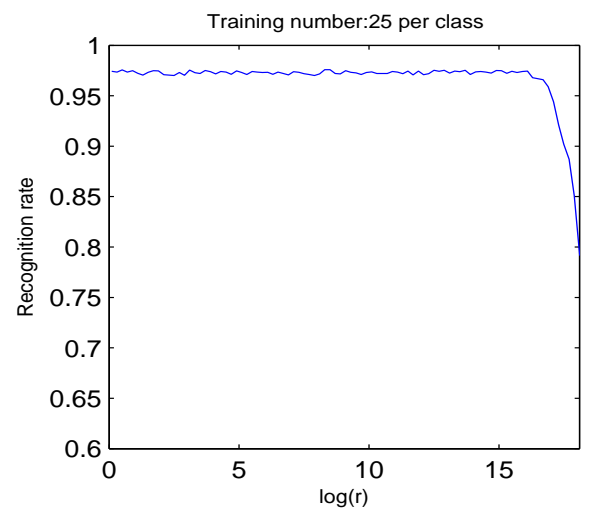

(b)

Figure 6: Recognition rate for the Iris database. (a) $0<r<100$; (b) $0<\log _{e}(r)<18$.

Table 7: Classification error probability for the Double-moon database.

\begin{tabular}{lccc}
\hline Algorithms & Minimum error & Maximum error & Mean error \\
\hline FLDA & 0.1523 & 0.2232 & 0.1763 \\
OFLD & 0.0166 & 0.0393 & 0.0288 \\
PNBDA & 0.1786 & 0.2642 & 0.1982 \\
FDNS & 0.0063 & 0.0395 & 0.0207 \\
OSNS & 0.0061 & 0.0242 & 0.0168 \\
SK-PCA & 0.0624 & 0.1256 & 0.0728 \\
FFS & 0.0714 & 0.1475 & 0.0984 \\
RSCDA & 0.0055 & 0.0186 & 0.0095 \\
\hline
\end{tabular}


Table 8: The average total CPU time (s) for each method on the Double-moon database.

\begin{tabular}{lcccccccc}
\hline Algorithms & FLDA & OFLD & PNBDA & FDNS & OSNS & SK-PCA & FFS & RSCDA \\
\hline Running time & 1.92 & 3.46 & 3.11 & 3.66 & 3.85 & 2.07 & 2.67 & 3.90 \\
\hline
\end{tabular}

Table 9: Recognition rate for the Double-moon database.

\begin{tabular}{lcccccccc}
\hline Algorithms & FLDA & OFLD & PNBDA & FDNS & OSNS & SK-PCA & FFS & RSCDA(average) \\
\hline Recognition rate & 0.8237 & 0.9712 & 0.8018 & 0.9793 & 0.9832 & 0.9272 & 0.9016 & 0.9903 \\
\hline
\end{tabular}

the separability of the Double-moon database. The number of training samples from each class is 50 and the rest are used for testing. As shown in Tables 7 and 8, RSCDA has the highest performance and CPU time among the eight algorithms. Moreover, we can see the impact of key parameter $r$ on the performance of the RSCDA in Fig 7(b) and the corresponding numerical recognition rates of related methods in Table 9 .

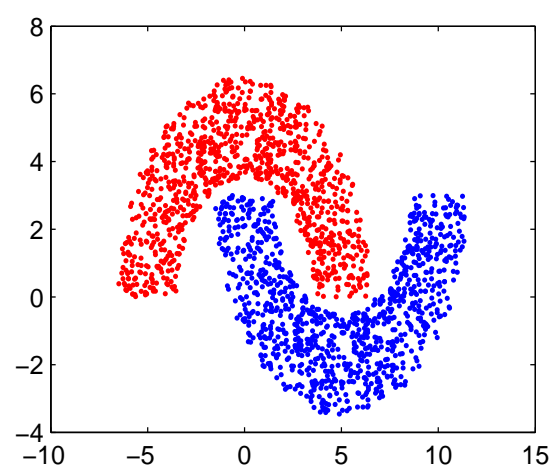

(a)

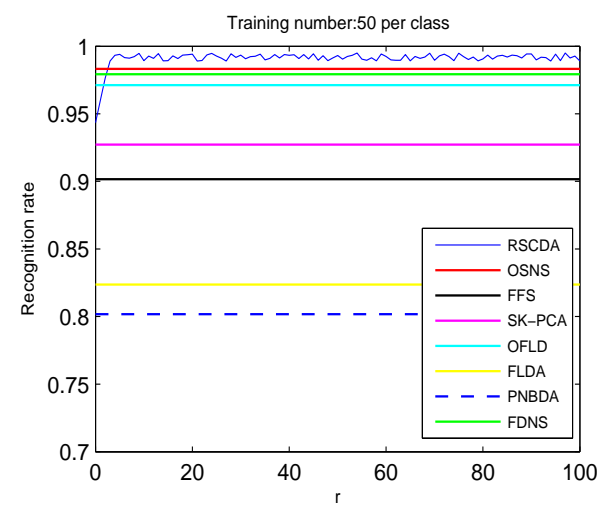

(b)

Figure 7: (a) Double-moon Database; (b) Recognition rate for the Double-moon database.

\subsection{Experiments with the Caltech-101 database}

The Caltech-101 dataset [33] consists of 9144 images from 101 object categories, including flowers, vehicles and animals. There is also an additional background class, making the total number of classes reach 102. There are about 30 to 800 images per category and most categories have about 50 images. Fig.8(a) shows the sample objects from the database. 20 samples were randomly selected from each class for the experiment. In order to improve the operating efficiency of computer, we convert original image to grayscale image and utilize bilinear interpolation method to resize it as $64 \times 64$ pixels. When the parameter is $r=8$, we compare RSCDA with several existing approaches (Table 10), and the optimal recognition rate is showed in Fig.9(a) and Table 11.

\subsection{Experiments with the COIL100 image database}

The COIL100 image database contains 7200 images of 100 objects viewed from varying angles at an interval of five degrees, resulting in 72 images per object. Fig.8(b) shows some sample objects from the database. Each image is first cropped around the object part(to remove background information) and we utilize bilinear interpolation method to resample it to a standard image size of 16 by 16 pixels. 20 samples from each class were randomly chosen for training for the experiment. When the parameter is $r=10$, we compare RSCDA with several existing approaches (Table 12), and the optimal recognition rate is showed in Fig.9(b) and Table 13.

The experimental results on different databases demonstrate that the proposed method achieves high recognition performance. One reason why RSCDA outperforms other methods is that distance information is considered in the mapping process of samples and angle information between samples is utilized in the 


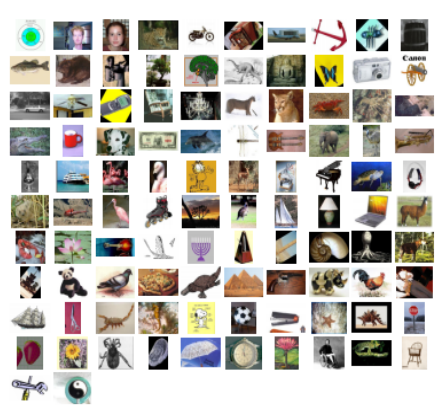

(a)

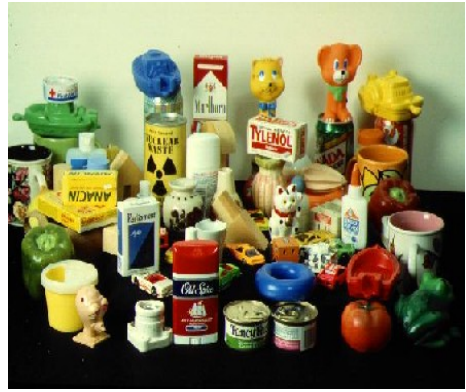

(b)

Figure 8: (a) Sample objects from Caltech-101 database; (b) Sample objects from COIL100 database.

Table 10: Classification error probability for the Caltech-101 database.

\begin{tabular}{lccc}
\hline Algorithms & Minimum error & Maximum error & Mean error \\
\hline OFLD & 0.5923 & 0.7241 & 0.6225 \\
PNBDA & 0.5643 & 0.7205 & 0.6127 \\
FDNS & 0.5485 & 0.6887 & 0.5920 \\
OSNS & 0.5309 & 0.6942 & 0.5822 \\
SK-PCA & 0.6066 & 0.7028 & 0.6633 \\
FFS & 0.5754 & 0.6933 & 0.6322 \\
RSCDA & 0.5523 & 0.6804 & 0.5912 \\
\hline
\end{tabular}

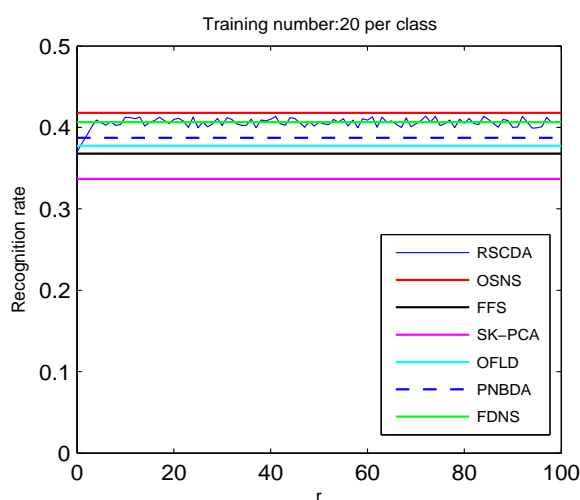

(a)

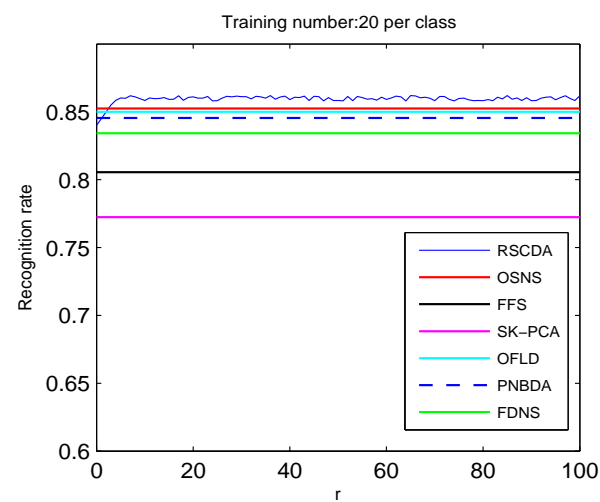

(b)

Figure 9: (a) Recognition rate for the Caltech-101 database; (b) Recognition rate for the COIL100 database.

Table 11: Recognition rate for the Caltech-101 database.

\begin{tabular}{lccccccc}
\hline Algorithms & OFLD & PNBDA & FDNS & OSNS & SK-PCA & FFS & RSCDA(average) \\
\hline Recognition rate & 0.3775 & 0.3873 & 0.4080 & 0.4178 & 0.3367 & 0.3678 & 0.4086 \\
\hline
\end{tabular}


Table 12: Classification error probability for the COIL100 image database.

\begin{tabular}{lccc}
\hline Algorithms & Minimum error & Maximum error & Mean error \\
\hline OFLD & 0.1322 & 0.1645 & 0.1490 \\
PNBDA & 0.1385 & 0.1688 & 0.1535 \\
FDNS & 0.1442 & 0.1868 & 0.1658 \\
OSNS & 0.1330 & 0.1645 & 0.1475 \\
SK-PCA & 0.1848 & 0.2882 & 0.2276 \\
FFS & 0.1624 & 0.2365 & 0.1945 \\
RSCDA & 0.1324 & 0.1625 & 0.1420 \\
\hline
\end{tabular}

Table 13: Recognition rate for the COIL100 image database.

\begin{tabular}{lccccccc}
\hline Algorithms & OFLD & PNBDA & FDNS & OSNS & SK-PCA & FFS & RSCDA(average) \\
\hline Recognition rate & 0.8510 & 0.8465 & 0.8324 & 0.8525 & 0.7724 & 0.8055 & 0.8580 \\
\hline
\end{tabular}

process of pattern recognition. Moreover, it can maintain the structural characteristics and separability of samples in the space transformation process.

\section{Conclusion}

A range space super spherical cap discriminant analysis method is put forward for the separability problem. The proposed method utilizes the normalized vectors in the surface of the unit spherical cap to denote the structural information of samples and some special natures of our model are proved. The method has no special requirement for the number of samples. If the dimension of the sample space is larger than the number of samples, the projection of the range space of $S_{T}$ can reduce the sample space to a low-dimensional space.

RSCDA with the special classifier is mainly applied to pattern recognition problems where different classes are located on different low-dimensional subspaces. Not only samples of known classes can be classified, but a new test sample that is not included in known classes, can also be found by our classifier. There is a key parameter $r$ that needs to be determined for our algorithm. Although the experiments show that the recognition rate is robust to the change of parameter $r$, the question of "How to find the most optimal parameter $r$ ?" needs to be deeply researched in the future.

\section{Appendix}

\section{Symbols used in this paper}

$R^{n}, V_{T}^{\perp}, C_{V_{T}^{\perp}}$ : initial space, range space, unit super superical cap space.

$\mathbf{S}_{B}, \mathbf{S}_{T}$ : Between-class scatter matrix and total scatter matrix of data population in initial space.

$\overline{\mathbf{S}}_{B}, \overline{\mathbf{S}}_{T}$ : Between-class scatter matrix and total scatter matrix of data population in range space.

$\hat{\mathbf{S}}_{B}, \hat{\mathbf{S}}_{T}$ : Between-class scatter matrix and total scatter matrix of data population in unit super Spherical cap space.

$\mathbf{x}, \overline{\mathbf{x}}, \hat{\mathbf{x}}$ : The sample in initial space, range space, unit super superical cap space, respectively.

$\mu_{0}, \bar{\mu}_{0}, \hat{\mu}_{0}$ : Total mean of data population in initial space, range space, unit superical cap space, respectively.

$\mu_{i}, \bar{\mu}_{i}, \hat{\mu}_{i}$ : The mean of class $i$ in initial space, range space, unit superical cap space, respectively.

$\|\bullet\|$ : The Euclidean norm of a vector or matrix.

$\operatorname{tr}(\bullet)$ : The trace of a matrix. 


\section{Acknowledgements}

The authors thank the support provided by the National Science Foundation of China (Grant No. 61331021, No. U1301251), and the Shenzhen Science and Technology Plan Project (JCYJ20130408173025036).

\section{References}

[1] R.A.Fisher, The use of multiple measurements in taxonomic problems, Annals of eugenics. 7 (1936) 179-188.

[2] D.C.Tao, X.L.Li, X.D.Wu, and S.J.Maybank, Geometric Mean for Subspance Selection, IEEE Trans. Pattern Anal. Machine Intell. 31 (2) (2009) 260-274.

[3] N. Gkalelis, V. Mezaris, and I. Kompatsiaris, Mixture Subclass Discriminant Analysis, IEEE Signal Processing letters. 18 (5) (2011) 319-322.

[4] Y.Cui, L.Fan, Feature extraction using fuzzy maximum margin criterion, Neurocomputing 86 (2012) $52-58$.

[5] H. Mohammadzade, D.Hatzinakos, Projection into expression subspaces for face recognition from single sample perperson,IEEE Trans.Affect.Comput. 4 (1) (2013) 69-82.

[6] Q. Gao, J.Liu, K.Cui, H.Zhang, X.Wang, Stable locality sensitive discriminant analysis for image recognition, NeuralNetw. 54 (2014) 49-56.

[7] Fukui, Kazuhiro, Maki, Atsuto, Difference subspace and its generalization subspace-based methods, IEEE Trans. Pattern Anal. Machine Intell. 37 (11) (2015) 2164-2177.

[8] Q.X.Gao, Q.Q.Wang, Y.F.Wang, X.B.Gao, X.Hong, H.L.Zhang, Dimensionality Reduction by Integrating Sparse Representation and Fisher Criterion and Its Applications, IEEE Trans on Image Processing. 24 (12) (2015) 5684-5695.

[9] Q.X.Cao, J.J.Liu, H.J.Zhang, J.Hou, X.J.Yang, Enhanced fisher discriminant criterion for image recognition, Pattern Recognit. 45 (10) (2012) 3717-3724.

[10] D.Zhang, J.Z.He, Y.Zhao, Z.L.Luo, M.H.Du, Global plus local: A complete framework for feature extraction and recognition, Pattern Recognit. 47 (3) (2014) 1433-1442.

[11] Q.X.Cao, J.J.Liu, H.L.Zhang, X.B.Gao, Joint global and local structure discriminant analysis, IEEE Trans on Information Forensics and Security. 8 (4) (2013) 626-635.

[12] M.A.Turk, A.P.Pentland, Face recognition using eigenfaces, in:Proceedings of the IEEE Conference on Computer Vision and Pattern Recognition. 1991, pp.586-591.

[13] J.Yang, J.Y.Yang, An optimal FLD algorithm for facial feature extraction, SPIE Proc.Intelligent Robots and Computer Vision XX: Algorithms, techniques, and Active Vision, 2001, pp.438-444.

[14] J.Jin, B.Wang, L.M.Zhang, A Novel Approach Based on Fisher Discriminant Null Space for Decomposition of Mixed Pixels in Hyperspectral Imagery, IEEE Geoscience and Remote sensing Letters. 7 (4) (2010) 699-703.

[15] H.Cevikalp, M.Neamtu, M.Wilkers, A.Barkana, Discriminative common vectors for face recognition, IEEE Trans. Pattern Anal. Machine Intell. 27 (1) (2005) 4-13.

[16] N.Vaswani, R.Chellappa, Principal components null space analysis for image and video classification, IEEE Trans on Image Processing. 15 (7) (2006) 1816-1830.

[17] L. J.Pu, W. X. Xie, J.H.Pei, A weak component approach of subspace analysis, Pattern Recognition and Computer Vision, MIPPR, GuiLin, China, 2011.

[18] M.L. Zhu, A. M. Martinez, Pruning noisy bases in discriminant analysis, IEEE Trans. Neural Netw. 19 (1) (2008) $148-157$.

[19] J.Yang, D.Zhang, J.Y.Yang, A generalized K-L expansion method which can deal with small sample size and highdimensional problems, Pattern Analysis Applications. 6 (1) (2003) 47-54.

[20] K. Liu, Y.Q. Cheng, J. Y. Yang, and X. Liu, An efficient algorithm for Foley-Sammon optimal set of discriminant vectors by algebraic method, Pattern Recognition and Articicial Intelligence. 6 (5) (1992) 817-829.

[21] H.Yu and J. Yang, A Direct LDA algorithm for high dimension data-with application to face recognition, Pattern Recognit. 34 (10) (2001) 2607-2070.

[22] L.F.Chen, H.Y.M.Liao, J.C.Lin, M.D.Kao, and G.J.Yu, A new LDA-based face recognition system which can solve the small sample size problem, Pattern Recognit. 33 (10) (2000) 1713-1726.

[23] Alok Sharma, Kuldip K. Paliwal, A two-stage linear discriminant analysis for face-recognition, Pattern Recognit.Lett. 33 (9) (2012) 1157-1162.

[24] X.N. Song, J.Y.Yang, X.J.Wu, X.B.Yang, An optimal symmetrical null space criterion of Fisher discriminant for feature extraction and recognition, Soft Computing. 15 (2) (2011) 281-293.

[25] W.S.Chen, X.L.Dai, B.B.Pan, T.Q.Huang, A novel discriminant criterion based on feature fusion strategy for face recognition, Neurocomputing 159 (2015) 67-77.

[26] Y.Zhao, W.S.Chen, B.B.Pan, B.Chen, Supervised kernel construction for unsupervised PCA on face recognition, in: The Chinese Conference on Pattern Recognition. 2014, pp. 351-359.

[27] L.J.Pu, W.X.Xie, J.H.Pei, Pattern Discriminant Based on Hyperspherical Discriminant Analysis of a Range Space, Signal Processing. 29 (8) (2013) 933-941.(in Chinese).

[28] Richard O. Duda, Peter E. Hart David G. Stork, Pattern Classification, 2 nd Edition, John Wiley Sons, Inc, New York, 2001, pp47-49.

[29] K. Liu, Y.Cheng, J.Yang, A generalized optimal set of discriminant vectors, Pattern Recognit. 25 (7) (1992) $731-739$.

[30] K. Liu, Y.Q.Cheng, J.Y.Yang, X.Liu, An efficient algorithm for Foley-Sammon optimal set of discriminant vectors by algebraic method, Int.J.Pattern Recog. Artif.Intell. 6 (5) (1992) 817-829. 
[31] Anthony K. H. Tung, Xin Xu, Beng Chin Ooi, CURLER: finding and visualizing nonlinear correlation clusters, Proceedings of the 2005 ACM SIGMOD international conference on Management of data, ACM, New York, USA, 2005, pp.467-478.

[32] Simon Haykin, Neural networks and learning machines, Third ed., Pearson Academic Inc., Hamilton, 2008.

[33] L. Fei-Fei, R. Fergus, P. Perona, Learning generative visual models from few training examples: an incremental Bayesian approach tested on 101 object categories, Comput. Vis. Image Underst. 106 (1) (2007) 59-70.

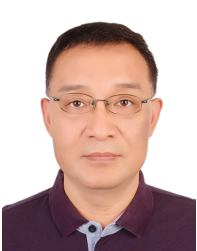

Jihong Pei was born in 1966. He received the B.S. degree in electronics engineering from Beihang University in 1989, and the M.S. degree in physical electronics and optoelectronics and the Ph.D. degree in signal and information processing from Xidian University, in 1994 and 1998, respectively. He is a Professor with Shenzhen University, and the Director of the Electronics Engineering Department with CIE, Shenzhen University. His research interests include intelligent information processing, and video and image analysis. He is a member of IEEE, and also a member of the Technical Committee of Information Fusion in the Chinese Society of Aeronautics and Astronautics.

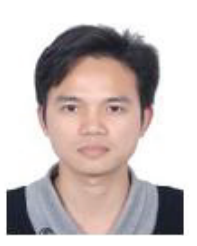

Hongguang Fan was born in 1983. He received the B.S. degree in applied mathematics from Neijiang Normal University, China, in 2006. He is currently pursuing the Ph.D. degree with the college of Information Engineering, Shenzhen University, China. His current research interests include signal analysis, pattern recognition and control theory.

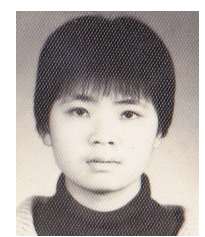

Lijuan Pu was born in 1981. She received the B.S. degree in electrical engineering from Guangdong Ocean University, China, in 2003, the M.S degree in signal and information processing from Shenzhen University, China, in 2006 and the Ph.D degree in the institute of Electronic Engineering from Xidian University, in 2013. Her current research interests include signal analysis and pattern recognition. 